\title{
Numerical investigations of blade shape effects on the flow characteristics in a stirred dead-end membrane bioreactor
}

\author{
Xu-Qu HU ${ }^{1, *}$, Xing-Yi WANG ${ }^{1}$, Xiu-Cheng LEI $^{1}$, Xiang QIU ${ }^{2}$, Lin-Feng CHEN ${ }^{3, \dagger}$ \\ 1. State Key Laboratory of Advanced Design and Manufacturing for Vehicle Body, \\ College of Mechanical and Vehicle Engineering, Hunan University, \\ Changsha 410082, PR China \\ 2. College of Science, Shanghai Institute of Technology, \\ Shanghai 201418, PR China \\ 3. Aerospace Engineering, Delft University of Technology, \\ Kluyverweg 1 Delft, 2629 HS, Netherlands
}

\begin{abstract}
Numerical simulations of turbulent flows in a stirred dead-end membrane bioreactor are performed by the RNG $k-\varepsilon$ model based on finite volume method using Fluent codes. Comparisons of numerical and experimental results confirm the reliability and feasibility of the constructed model. Flow structures such as wake flows and circulation loops in stirred flows were well simulated. An increase of stirring speed is proposed to use to minimize the low velocity region. The single vane stirrer is found to be beneficial for biological separations. Results reveal that the increase of vane number can enhance the mixing effect in flow domains. However, a circular disk stirrer goes against the formation of vertical circulations. The six-vane stirrer is found to be able to provide a uniform distribution of high shear stress.
\end{abstract}

Key words: Turbulent flow, Stirred flows, Membrane fouling, Membrane bioreactor

\section{Introduction}

Membrane filtration has been widely applied in many industrial separation treatments, such as wastewater treatment and biological separation ${ }^{[1]}$. For biological separations, membrane bioreactors (MBR), which consists of a submersed micro-porous membrane to filtrate feed solution by forcing it to permeate through membranes, is considered as one of the most efficient apparatuses ${ }^{[2]}$. As suspending materials are inevitable to deposit on membrane surface, either pore blockage or cake-layer formation on membrane surface can lead to significant flux decline and rapid membrane degradation ${ }^{[3]}$. The effect of membrane fouling is a significant problem for the widespread use of membrane filtration techniques. During the past several decades, many strategies were proposed to control membrane fouling, such as membrane washing and cleaning $^{[4]}$, gas sparging ${ }^{[5]}$ and dynamic filtration ${ }^{[6]}$.

\footnotetext{
${ }^{*}$ Co-corresponding author, E-mail: huxuqu@gmail.com

$\dagger$ Corresponding author, E-mail: Lnfngchn@gmail.com
}

Dynamic filtration, also known as shear-enhanced filtration, consists in altering hydrodynamic characteristics and producing high shear rates by a moving part near the fixed membrane in container ${ }^{[7]}$. Comparing with conventional cross flow filtrations, dynamic filtration is usually operated at moderate transmembrane pressure (TMP) and small inlet flow rate. Its total cost is normally lower than that of conventional techniques, since the specific hydrodynamic characteristics in dynamic filtration systems is beneficial for energy conservation, membrane fouling control and investment reduction ${ }^{[8]}$. An increasing number of researchers have paid their attention to examine the hydrodynamic characteristics and membrane fouling mechanism in dynamic filtration systems using experimental and numerical techniques, e.g., in Ref. [6]. Lee et al. have utilized a rotating solid disk to create high shear rates in dynamic filtration bioreactors to concentrate recombinant yeast cells ${ }^{[9]}$. The stirring speed was found to be one of the key factors for flow characteristics in reactors. Jiang et al. have experimentally studied the relationship between rotating speed and membrane fouling using particle 
(a)

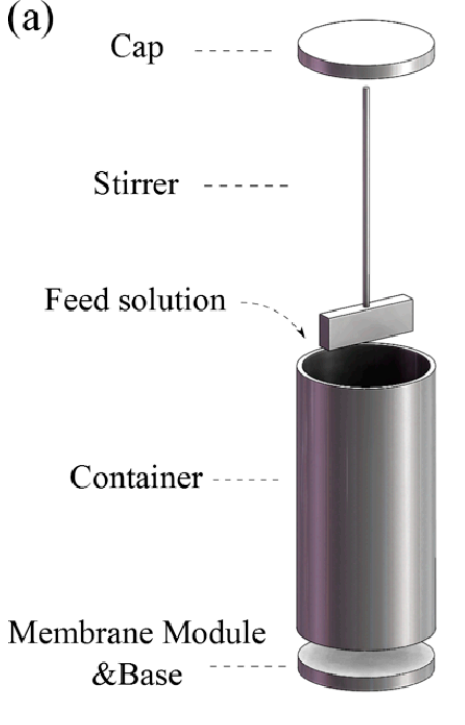

(b)

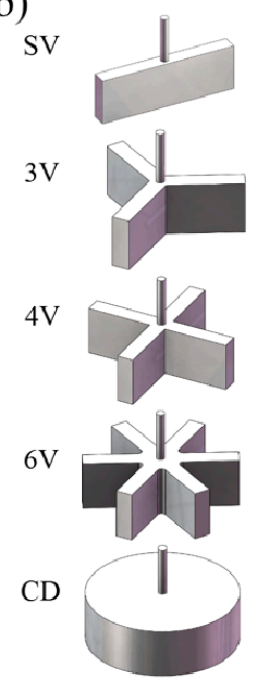

(c)

Global mesh

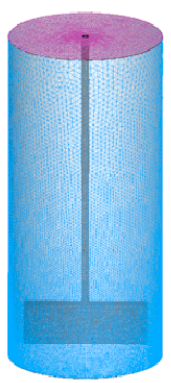

Mesh on membrane surface

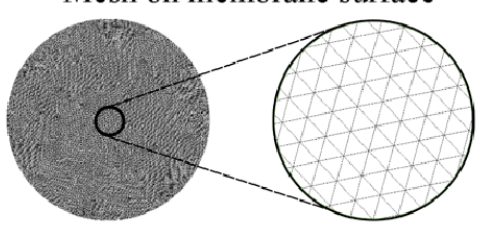

Fig. 1. Schematic of an enzymatic membrane reactor. (a) Exploded drawing of device.

(b) Different types of stirring blades. (c) Corresponding mesh.

image velocimetry ${ }^{[10]}$. They claimed that the increase of rotating speed is an effective and energy-saving method for preventing membrane fouling, as it could induce intensive turbulent flow in reactor. Torras et al. have numerically studied the shear stress distribution on membrane surface in rotating disk filtration modules ${ }^{[11,}$ 12]. The results obtained by commercial code Fluent coincide very well with experimental data, which implies that numerical techniques are suitable for hydrodynamic studies in dynamic filtration systems. Parvareh et al. have applied the renormalization group (RNG) $k-\varepsilon$ model to consider turbulent flow in a microfiltration module with marine type impellers, in which the permeate flux is found to be highly relevant with flow characteristics and dynamic pressure distribution on membrane surface ${ }^{[13]}$.

The stirring blade shape is not only highly relevant with the generated flow characteristics in membrane reactors, but it can also determine the optimal operating conditions in dynamic filtration process. Brou et al. have creatively constructed experimental equipment for microfiltration of yeast suspensions by integrating vanes to rotating disk. They found that the introduction of vanes to disk surface can generate high permeate flux and prevent membrane fouling, as it enhances the mixing effect by generating more vortices near the membrane surface ${ }^{[14]}$. Sen et al. have studied the vane geometry effect on shear stress distribution on membrane surface in a rotating disk module, in which the maximum permeate flux was found to be achieved by $45^{\circ}$ angle perforated vanes ${ }^{[15]}$. Hwang et al. have focused on the shape effect of rotating disk (e.g., vane number) on flow characteristics in a microalgae microfiltration system. The filtration performance is found to be intensively affected by the disk structure and rotation speed ${ }^{[16]}$. Kim et al. have numerically studied the flow domain generated by perforated or unperforated disks in a dynamic filtration system to harvest microalgae, which is confirmed to generate high shear rates on the membrane comparing to the device with an unperforated disk ${ }^{[17]}$. Sarkar et al. have designed a novel shear enhanced membrane module, which consists of a hollow basket with four radial arms and installs on the central shaft ${ }^{[18]}$. The numerical results indicate that the accumulated solute is dispersed from the membrane surface due to the intensive circulation flow generated by basket rotation. However, to our best knowledge, numerical studies about the blade shape effect on hydrodynamic characteristics in an enzymatic bioreactor are still not too many.

In this study, we focus on the blade shape effect on the hydrodynamic characteristics in a widely used enzymatic bioreactor. A three-dimensional numerical model is constructed to simulate the stirred turbulent flows in an enzymatic bioreactor equipped with membrane. The Computational Fluid Dynamics (CFD) Fluent codes were applied for numerical implementations. The features of stirred flows by a straight-vane stirrer are firstly analyzed. Careful discussions on the flow velocities, TMP and shear stress distribution are all well presented. The flow characteristics induced by different blades (with different vane number) at different stirring speeds are then examined. The collaborating effects of the blade 


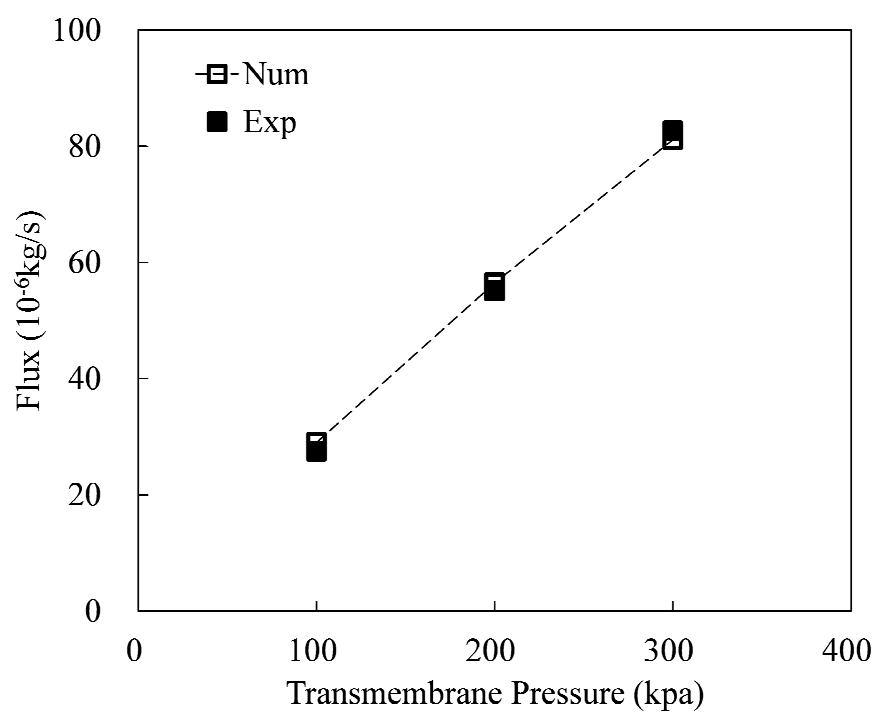

Fig. 2. Comparison of numerical and experimental results of flux to transmembrane pressure.

shapes and stirring speeds, on the shear stress distribution on the membrane surface, are also analyzed. An optimal situation is thus determined in terms of the highest and most-uniform distribution of shear stress on the membrane surface.

\section{Methods and models}

\subsection{Problem description}

We study the stirred flows in a dead-end enzymatic membrane bioreactor (Amicon 8050, Millipore, USA) that has been widely applied for separation treatments in bioengineering. As shown in Fig. 1 (a), the membrane module of a diameter of 45 $\mathrm{mm}$ is fixed at the bottom of a cylindrical container. Total height of the container is $98 \mathrm{~mm}$. A blade is mounted to the end of a straight shaft, which can be magnetically rotated to generate rotating flow and high shear stress near the membrane surface. The gap between membrane surface and the bottom of the blade is $4 \mathrm{~mm}$.

We consider the pure water, acting as the feed solution, is continuously pumped into container from the inlet. The feed solution is forced to flow towards the bottom of container under the pressure-driven effect. An ultrafiltration membrane (RC10-M, PLGC, Millipore, USA) is settled on the membrane module, which allows the pure water to penetrate through but obstructs large molecular particles inside the container in separation treatments. At the bottom of container, an array of blades is applied to generate high-speed rotation flows. We have considered five different types of stirring blades to investigate the effect of blade geometry on the hydrodynamic effects in this stirred flow. The blade shapes are demonstrated in Fig. 1 (b), which can be called by straight-vane stirrer (SV), threevane $(3 \mathrm{~V})$ stirrer, four-vane $(4 \mathrm{~V})$ stirrer, six-vane $(6 \mathrm{~V})$ stirrer and circular-disk (CD) stirrer, respectively. For $\mathrm{SV}, 3 \mathrm{~V}, 4 \mathrm{~V}$ and $6 \mathrm{~V}$ stirrers, the blade length and height are $36 \mathrm{~mm}$ and $12 \mathrm{~mm}$, and blade widths are all $4 \mathrm{~mm}$. The radius of CD stirrer is $36 \mathrm{~mm}$, and its height is 12 $\mathrm{mm}$. The blade rotation speed is assumed to be 150,200 and 400 rounds per minute (rpm).

\subsection{Numerical model}

The stirred flow is governed by Navier-Stokes equations,

$$
\begin{aligned}
& \vec{\nabla} \cdot(\rho \vec{u})=0, \\
& \frac{\partial}{\partial t}(\rho \vec{u})+(\vec{u} \cdot \vec{\nabla}) \cdot \vec{u}=\rho \vec{f}-\vec{\nabla} \cdot p+\mu \Delta \vec{u},
\end{aligned}
$$

where $\vec{u}$ denotes the velocity, $p$ is the pressure, $f$ is the body force and $\mu$ is the dynamic viscosity. As it is stirred by high rotating speeds, the fluid flow in this reactor is under a relatively high Reynolds number. The turbulent effect needs to be taken into account in our modeling. We apply the RNG $k-\varepsilon$ turbulence model, which is initially derived by Yakhot and Orszag from the renormalization group theory ${ }^{[19]}$. Although it has similar forms to that of standard $k-\varepsilon$ turbulence model, it considers the empirical nature of turbulent flow and modifies turbulent viscosity and dissipation rate equations to better describe rapidly strained flows, such as stirred flows ${ }^{[20]}$. The RNG $k-\varepsilon$ turbulence model has been widely used for turbulent flows ${ }^{[21]}$. The turbulent kinetic energy and dissipation rate equations can be expressed as,

$$
\begin{aligned}
& \frac{\partial}{\partial t}(\rho k)+\frac{\partial}{\partial x_{i}}\left(\rho k u_{i}\right) \\
& =\frac{\partial}{\partial x_{j}}\left(\alpha_{k} \mu_{e f f} \frac{\partial k}{\partial x_{j}}\right)+G_{k}-\rho \varepsilon
\end{aligned}
$$


(a) Vel. Mag. $(\mathrm{m} / \mathrm{s})$

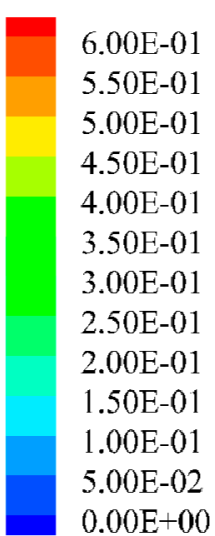

(b)

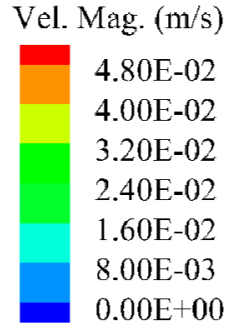

$400 \mathrm{rpm}$
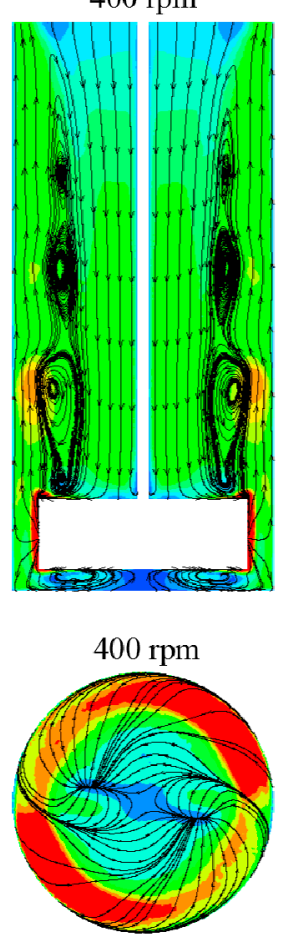

$250 \mathrm{rpm}$
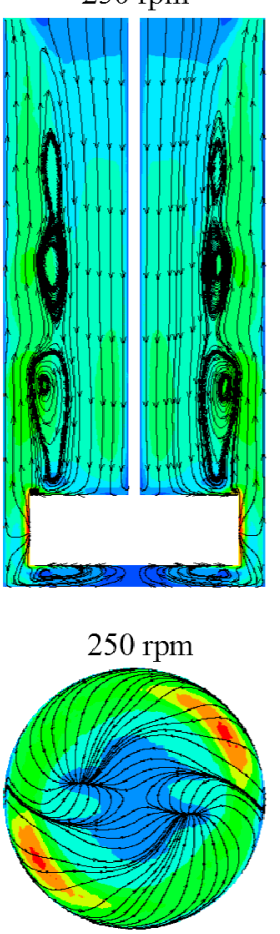

$150 \mathrm{rpm}$
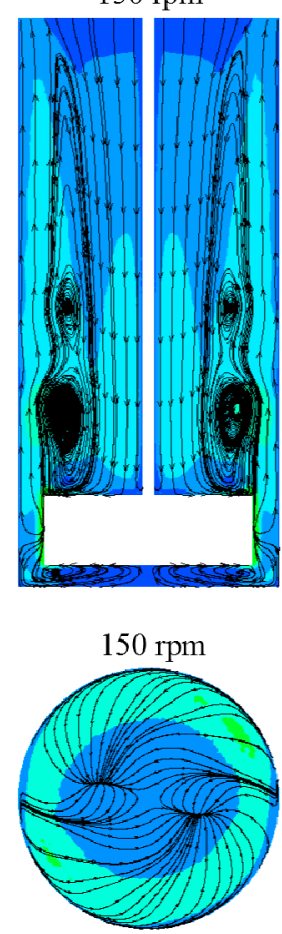

Fig. 3. Velocity distributions on vertical (a) and horizontal (b) planes in a stirred reactor.

$\frac{\partial}{\partial t}(\rho \varepsilon)+\frac{\partial}{\partial x_{i}}\left(\rho \varepsilon u_{i}\right)$

$=\frac{\partial}{\partial x_{j}}\left(\alpha_{\varepsilon} \mu_{e f f} \frac{\partial \varepsilon}{\partial x_{j}}\right)+C_{1 \varepsilon} \frac{\varepsilon}{k} G_{k}-C_{2 \varepsilon}^{*} \rho \frac{\varepsilon^{2}}{k}{ }^{\prime}$

where the modified viscosity $\mu_{\text {eff }}$ equals to the sum of dynamic viscosity $\mu$ and the eddy viscosity $\mu_{t}$, and $\mu_{t}$ can be expressed as $\mu_{t}=\rho C_{\mu} k^{2} / \varepsilon$. We use $G_{k}$ to represent the generation of turbulence kinetic energy due to the mean velocity gradients, which is written as, $G_{k}=\mu_{t} \rho\left(\frac{\partial u_{i}}{\partial x_{j}}+\frac{\partial u_{j}}{\partial x_{i}}\right) \frac{\partial u_{i}}{\partial x_{j}}$,

The coefficients occurred in these formulas are defined as $C_{1 \varepsilon}=1.42, C_{\mu}=0.0845, \alpha_{k}=\alpha_{\varepsilon}=1.39$. The other coefficient $C_{2 \varepsilon}^{*}$ is defined as,

$C_{2 \varepsilon}^{*}=C_{2 \varepsilon}+C_{\mu} \eta^{3} \frac{1-\eta / \eta_{0}}{1+\beta \eta^{3}}$,

where $C_{2 \varepsilon}=1.68, \eta_{0}=4.38, \beta=0.012$. The coefficient $\eta$ takes the form as $\eta=\sqrt{2 S_{i j} \cdot S_{i j}} \cdot k / \varepsilon$, where $S_{i j}$ denotes the strain tensor.

When viewed from the stationary frame, the stirred flows around shaft can be considered as unsteady flows. However, some approaches with a moving reference frame can be employed to simplify the flow around the moving part as a steady-state problem, such as single reference frame (SRF) and multiple reference frame (MRF) approaches ${ }^{[22]}$. The accuracy and reliability of SRF and MRF for rotating flows have been validated by several researchers, e.g., Yataghene et al. ${ }^{[23]}$ and $\mathrm{Hu}$ et al. ${ }^{[24]}$. The SRF approach is applied in present study.

\subsection{Numerical implementation}

The CFD codes Fluent 15.0 is applied to perform the numerical simulations of stirred flow in the reactor. It is based on the finite volume method, in which the flow domain is discretized into a finite set of control volumes. The pressure-based and implicit numerical solver is applied in the simulations. The SIMPLE algorithm is used in pressure velocity coupling process. A constant pressure of $200 \mathrm{kPa}$ is applied at the inlet, while the atmospheric pressure is at the outlet. The physical behavior of RC10-M membrane is considered equivalent to a porous media, which allows the water to pass through. The effect of membrane resistance is described by Darcy's law, for which the resistance coefficient is determined from our experimental results. As shown in Fig. 1 (c), the flow domain is discretized by an unstructured tetrahedral mesh with about $3.1 \times 10^{6}$ unites. In order to achieve high accuracy at a relatively low computational cost, a fine mesh of characteristic length about $0.3 \mathrm{~mm}$ is used for the membrane domain and that near the blade surfaces, while a coarse one of 

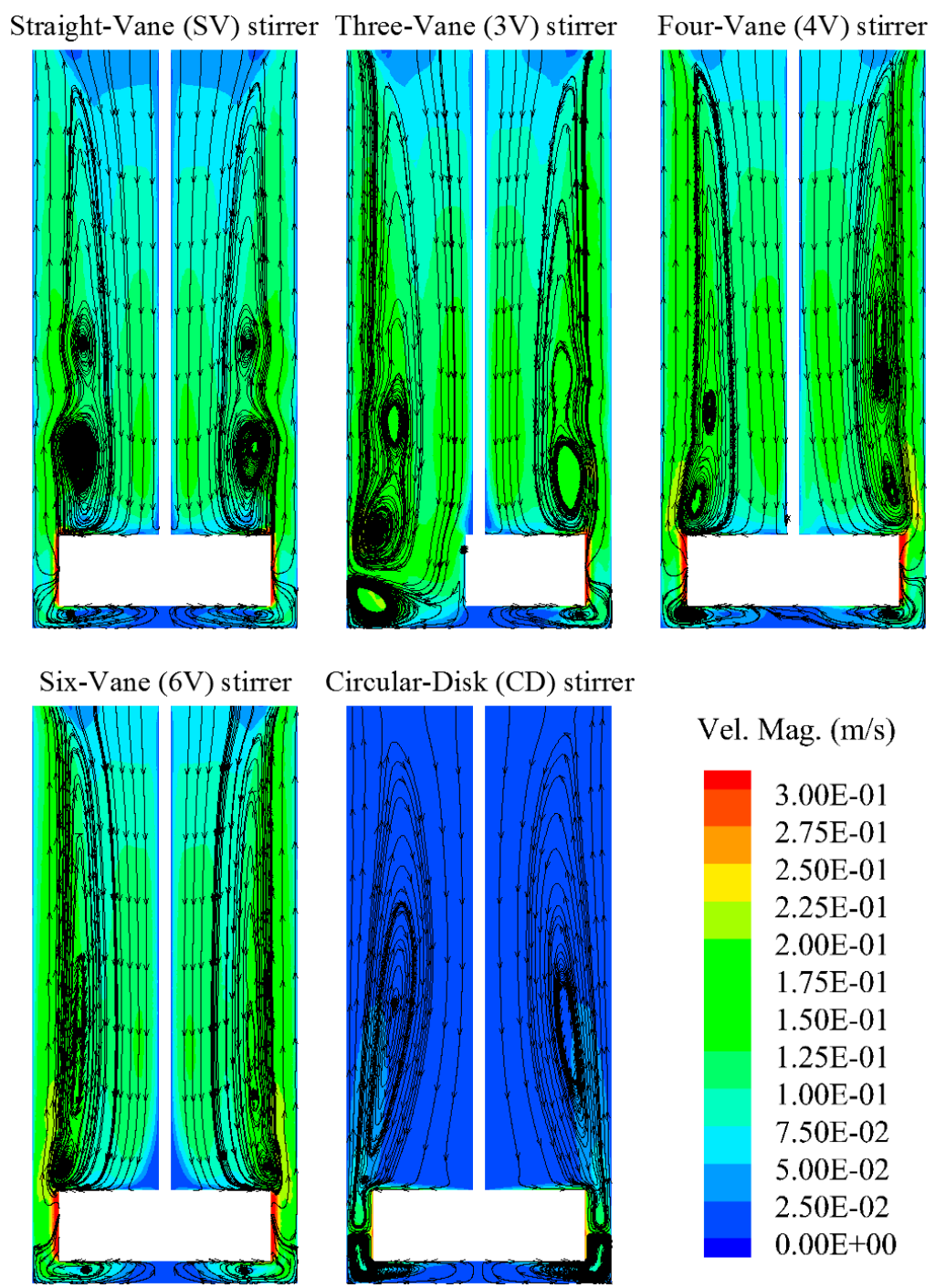

Circular-Disk (CD) stirrer

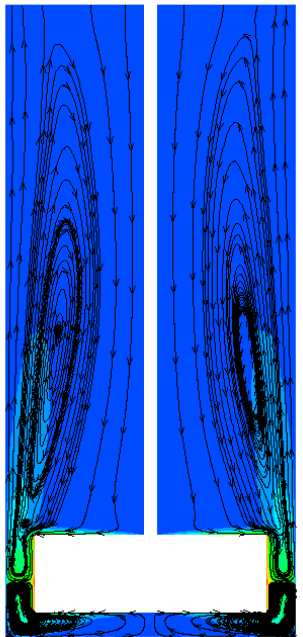

Vel. Mag. $(\mathrm{m} / \mathrm{s})$

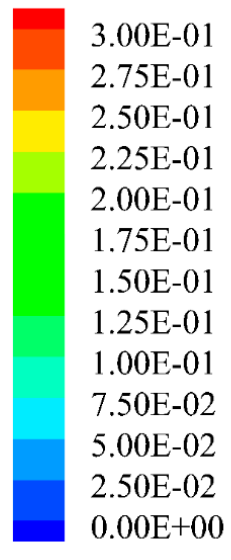

Fig. 4. Velocity distributions on vertical planes with five geometries of stirring blade at $150 \mathrm{rpm}$.

about $1.7 \mathrm{~mm}$ is applied in the region close to the inlet. The influence of mesh size has been validated using a twice-smaller mesh, with which there is no significant difference observed in between.

\subsection{Validation}

An experimental investigation of the relationship between the flux and TMP was reported in the same reactor $^{[25]}$, which found that the flux increased linearly with the TMP for the RC10-M membrane. It means that the membrane filtration resistance is constant, which is adopted to be a reference data for the determinations of physical parameters of the porous media (membrane) in present numerical model. The variations of flux with respect to TMP, obtained from experimental or numerical approaches, are as shown in Fig. 2. It shows that the experimental and numerical results coincide well with each other, which validates the reliability and feasibility of the proposed numerical models.

\section{Results and discussions}

The fluid velocity and shear stress near the membrane surface are important for the transportation and deposition of pollution materials in MBR, and consequently are critical factors in preventing and controlling of membrane fouling. A typical technique to generate flow fields as required is to apply a rotating module (e.g., straight-vane stirrer or perforated disk) near the membrane surface in filtration systems ${ }^{[15-17]}$. The hydrodynamic characteristics of stirred flows depend largely on the blade stirring speed. The internal flows in a dead-end membrane reactor driven by a straight-vane stirrer at different rotation speeds were therefore simulated by our constructed models. 

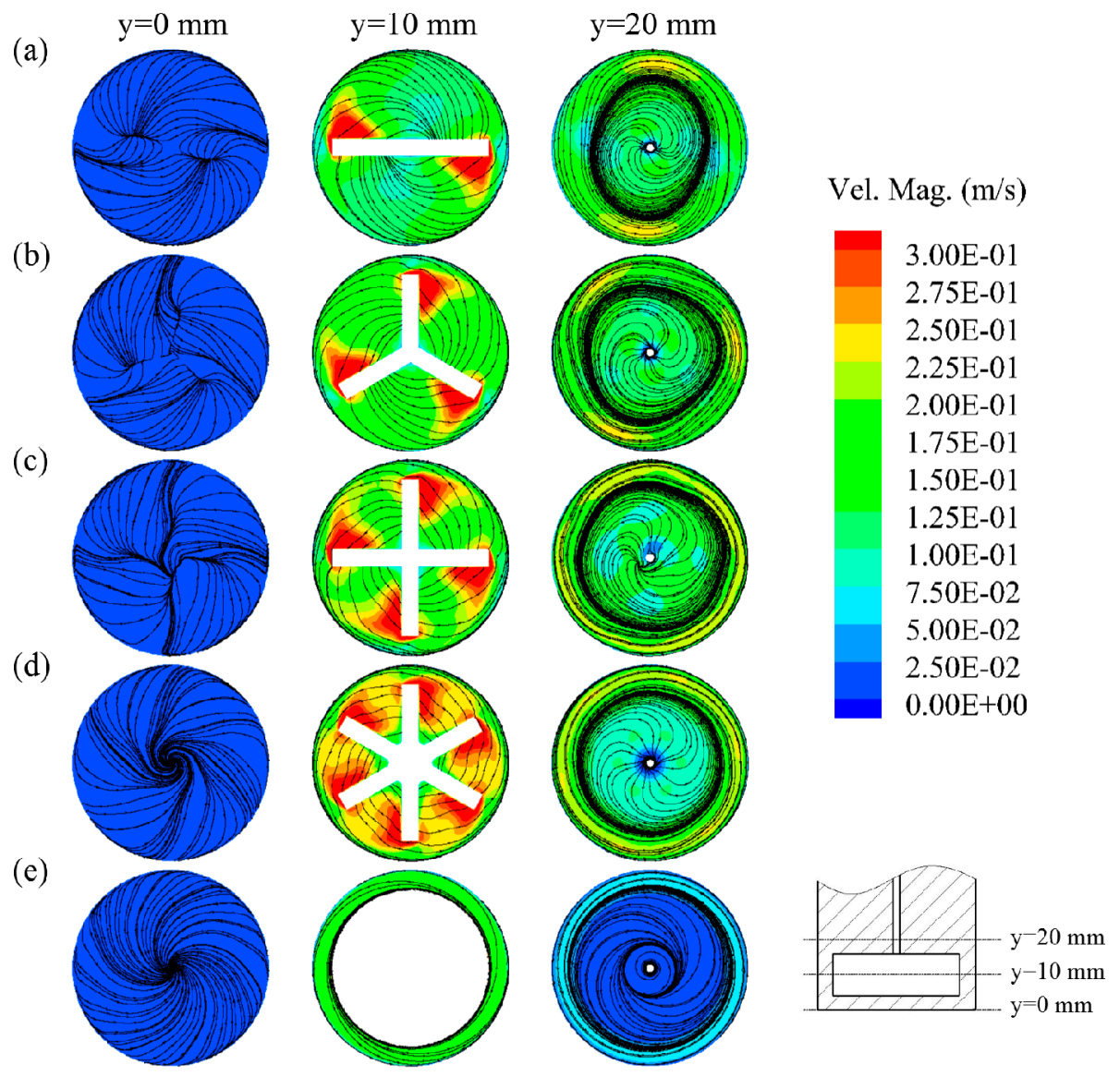

Fig. 5. Distributions of flow velocities at $150 \mathrm{rpm}$ on different horizontal planes.

Fig. 3 (a) and (b) respectively show the fluid velocity distributions in vertical and horizontal planes, for which the stirring speed of the straight-vane stirrer is 150,250 and $400 \mathrm{rpm}$ from left to right. It can be noted in Fig. 3 (a) that, the internal fluid is driven by the radial discharge from blade to rotate and flow centrifugally towards the container wall. The centrifugal jet splits into two main tributary flows near the small gap between the blade tip and the sidewall of the container, with the fluid velocity being higher near the blade tips than that in the central part of the reactor. One upward spiral flow migrates towards the inlet, and the other downward spiral flow rotates near the membrane surface. A strong circulation loop extending over a large volume from the inlet to the top of the blade can be found around the shaft, which is mainly formed by the merging of the upward spiral flow and the inflow of feed-water. These flow patterns have also been revealed by many previous studies using experimental and/or numerical techniques on other dynamic filtration systems of different types of stirrers $^{[13,22]}$. As the stirring speed increases from 150 to 250 and to $400 \mathrm{rpm}$, the internal flow can be apparently accelerated as the overall color distributions of the fluid velocity become lighter and brighter (Fig. 3). Since high fluid velocity is beneficial for the preventing of membrane fouling, our results coincide with the practical experience that the increase of blade stirring speed is an effective strategy to improve the membrane performance $e^{[9,10]}$. However, a low velocity region in light blue can be always found in Fig. 3 (b) at the regional center of membrane surface, despite that it shrinks as the stirring speed increases. As the membrane fouling would likely accumulate at the region of low fluid velocity, a better design of blade geometry to reduce the low velocity region area on the membrane surface is thus necessary for the improvement of efficient use of the membrane, especially for those biological filtrations that are only allowed to be operated at low stirring speeds.

Considering a low stirring speed of $150 \mathrm{rpm}$, the internal flows induced by different shaped stirrers were simulated by the proposed model. Fig. 4 shows the distributions of fluid velocity on vertical planes, for which the stirrer shapes are straight-vane (SV), threevane $(3 \mathrm{~V})$, four-vane $(4 \mathrm{~V})$, six-vane $(6 \mathrm{~V})$ and circular- 


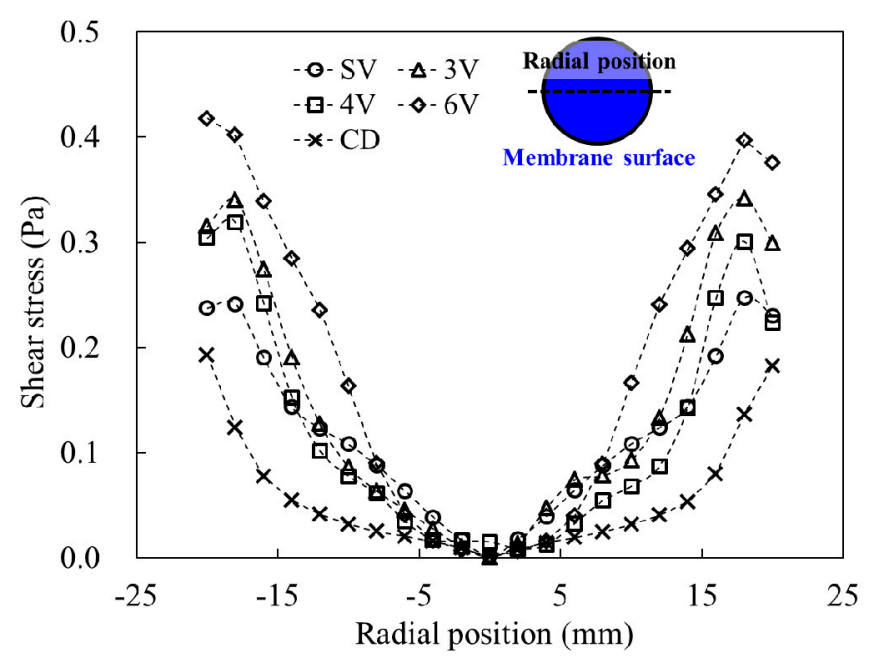

Fig. 6. Distribution of shear stress along the radial line on membrane surface at $150 \mathrm{rpm}$.

disk (CD), respectively, see Fig. 1 (b). The circulation loops that are merged by the upward spiral flow and the feed-water inflow can be found for all stirrers. The top ring vortices inside the circulation loops are almost symmetric about the shaft for the SV case with a almost horizontal centrifugal flow jet, which is in contrast with that of the $3 \mathrm{~V}$ case where the top ring vortices on the left side are inclined towards the membrane surface with a relatively large vortex persisting at the bottom left corner of the container. This difference primarily comes from the asymmetric arrangement of vanes of $3 \mathrm{~V}$ stirrer on horizontal plane. As the number of vanes increases from 1 to 6 , the area of high flow velocity near the blade tips becomes larger and thicker, but no significant changes in the overall color of fluid velocity can be found. It implies that increasing the vane number is not an effective way to accelerate the overall internal flow fields. However, the vortex structure varies significantly with the increase of vane number. The layered ring vortices on top of the blade are found to merge gradually as the stirrer type varies from SV to $3 \mathrm{~V}$ and to $4 \mathrm{~V}$, and finally expand over almost all of the circulation loops for the $6 \mathrm{~V}$ stirrer. This result indicates that the flow mixing effect can be enhanced if more vanes are applied. For the CD stirrer, it is of interest to notice that the flow domain is separated to two parts: the upper circulation loops above the stirrer and the lower vortex flow rotating near the membrane surface. The vertical convection flow is almost completely restrained by the $\mathrm{CD}$ stirrer, and the overall flow velocity is therefore obviously lower than that of other stirrers.

Fig. 5 shows the distributions of fluid velocity on different horizontal planes with a counter-clockwise stirring speed of $150 \mathrm{rpm}$. The structure of upward/downward flow is more apparent on horizontal views. Taking the case of SV stirrer for example, see Fig. 5 (a), the fluid velocities on the membrane surface $(y=0 \mathrm{~mm})$ are very low as the downward spiral flow is decelerated under the effects of wall friction. On a horizontal plane that cuts the stirrer $(y=10 \mathrm{~mm})$, the wake flows can be clearly identified at the leeward side of the impeller blades, in which the fluid velocities are significantly higher than that of the other areas. An elliptical vortex can be found on the horizontal plane above the SV stirrer $(y=20 \mathrm{~mm})$, which is mainly created by the upward spiral flow. As the vane number increases, the vortex structure changes to a triangularlike shape for $3 \mathrm{~V}$ stirrer, a square-like shape for $4 \mathrm{~V}$ stirrer and a circular-like shape for $6 \mathrm{~V}$ stirrer. Moreover, the area of wake flows is also observed to expand with the increase of vane number, as it takes over almost all the peripheral area of the circular plane with a $6 \mathrm{~V}$ stirrer, as shown in Fig. 5 (d, middle). These results are in consistence with experimental observations that the increase of vane number can enhance the mixing effect of stirred flows. However, Fig. 5 (e) shows that the flow velocities induced by a CD stirrer are much lower than that of the others, even if the CD stirrer can be regarded as an extreme case of the increase of vane number. This divergence between the numerical results and physical deductions, are mainly caused by the restrained effect of mixing flows. As shown in Fig. 5 (e, middle), the circular disk that almost blocks the cylindrical reactor is not beneficial for the formation of vertical mixing flows.

The distributions of shear stress along a radial line on the membrane surface at $150 \mathrm{rpm}$ are presented in Fig. 6. The shear stresses are almost zero at the central area of the membrane, but it increases rapidly on the radical direction to the peripheral area of membrane. The maximum shear stress occurs near the marginal 


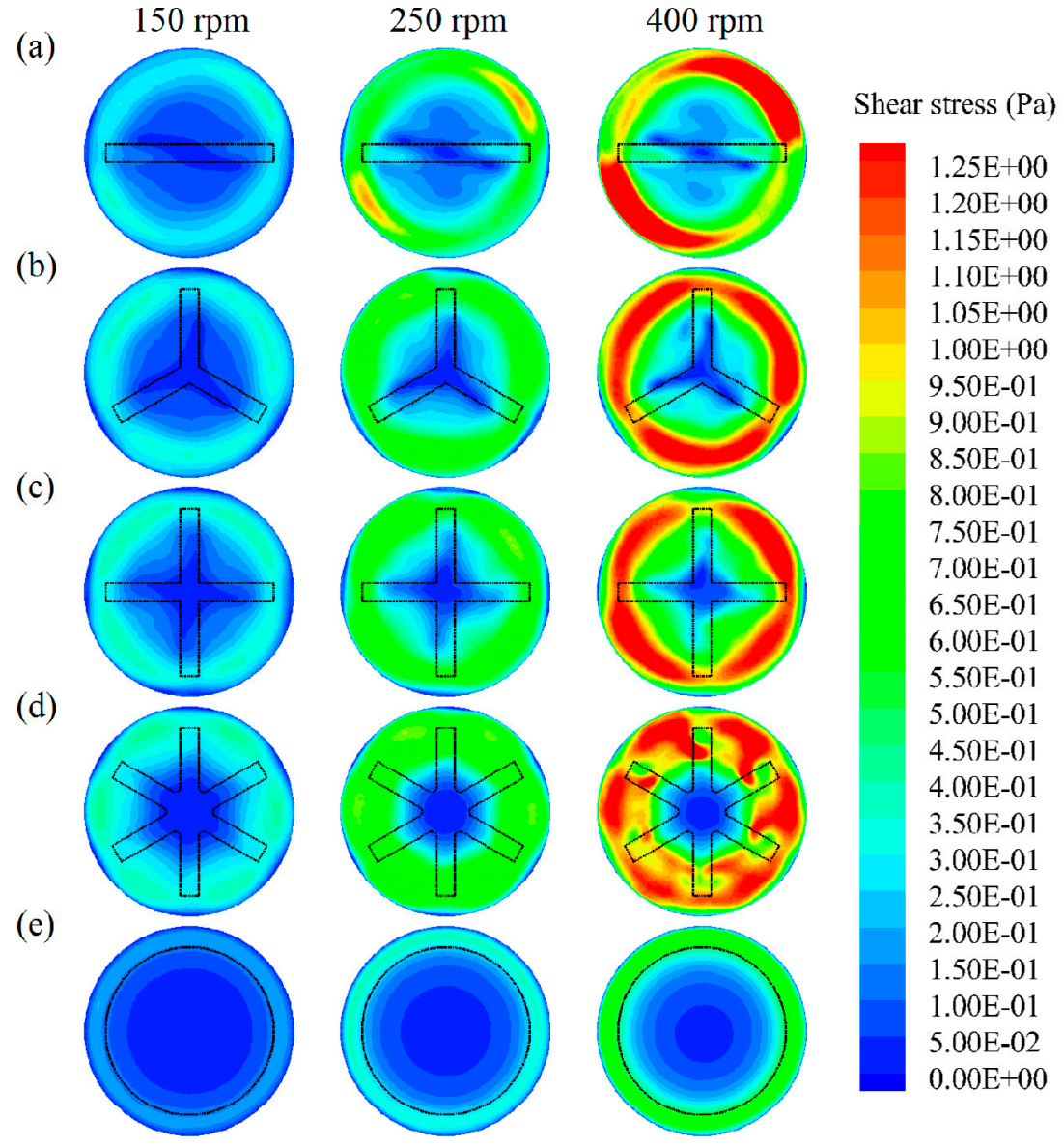

Fig. 7. Distribution of shear stress on membrane surface at different stirring speeds.

area of membrane. This non-uniform distribution of shear stress along the radial direction mainly results from the uneven distribution of flow velocities. A sharp decrease of the shear stress at the marginal area exists in most of these cases, which is caused by the complex flow behaviors at the gap between blade tip and container wall.

Fig. 7 shows shear stress patterns for different blade geometries at each stirring speed. Firstly, considering the increasing of the stirring speed, for each stirrer the effect of it on the shear stress is clear from group (a) to (e). As illustrated in Fig. 7 (a, left), the magnitude of shear stress on the membrane surface of SV stirrer at $150 \mathrm{rpm}$ stays at a low value ranging from 0 to $0.37 \mathrm{~Pa}$, which is due to the effect of wall friction as mentioned above. As shown in Fig. 7 (a, middle), because the downward spiral flow is strengthened by the increase of stirring speed, the magnitude of shear stress at the peripheral area of circular plane is apparently larger than at the central area. The maximum value approximately increases to $1.06 \mathrm{~Pa}$ at $250 \mathrm{rpm}$. As the stirring speed increases to $400 \mathrm{rpm}$, as shown in Fig. 7 (a, right), the shear stress at peripheral area becomes higher, and its value reaches about $2.13 \mathrm{~Pa}$. In addition, it can be seen that the downward spiral flow at peripheral area is dragged by blade tips and moves in a counter-clockwise direction. Secondly, as the vane number increases, it can be seen that these downward spiral flows are restrained in a specific area. Especially for the $6 \mathrm{~V}$ stirrer, they nearly hold all the peripheral area of the circular plane, as shown in Fig. 7 (d, right). Moreover, the shear stress distributions become increasingly uniform from SV to 6V stirrer. Specially, Fig. 7 (e) shows that the shear stress induced by a CD stirrer at all stirring speeds are lower than that of other stirrers. A possible reason for this result is the blocking effect on the formation of vertical mixing flows of this circular disk. From the analysis above, it can intuitively be indicated that fluid characteristics can be improved by increasing stirring speed and vane number.

Fig. 8 (a) shows the collaborating effects of the blade shapes and stirring speeds on the facet average of shear stress (also called mean shear stress) on the membrane surface. It shows that the increase of stirring speed results in a higher shear stress, which makes sense as the shear stress depends largely on the velocity 

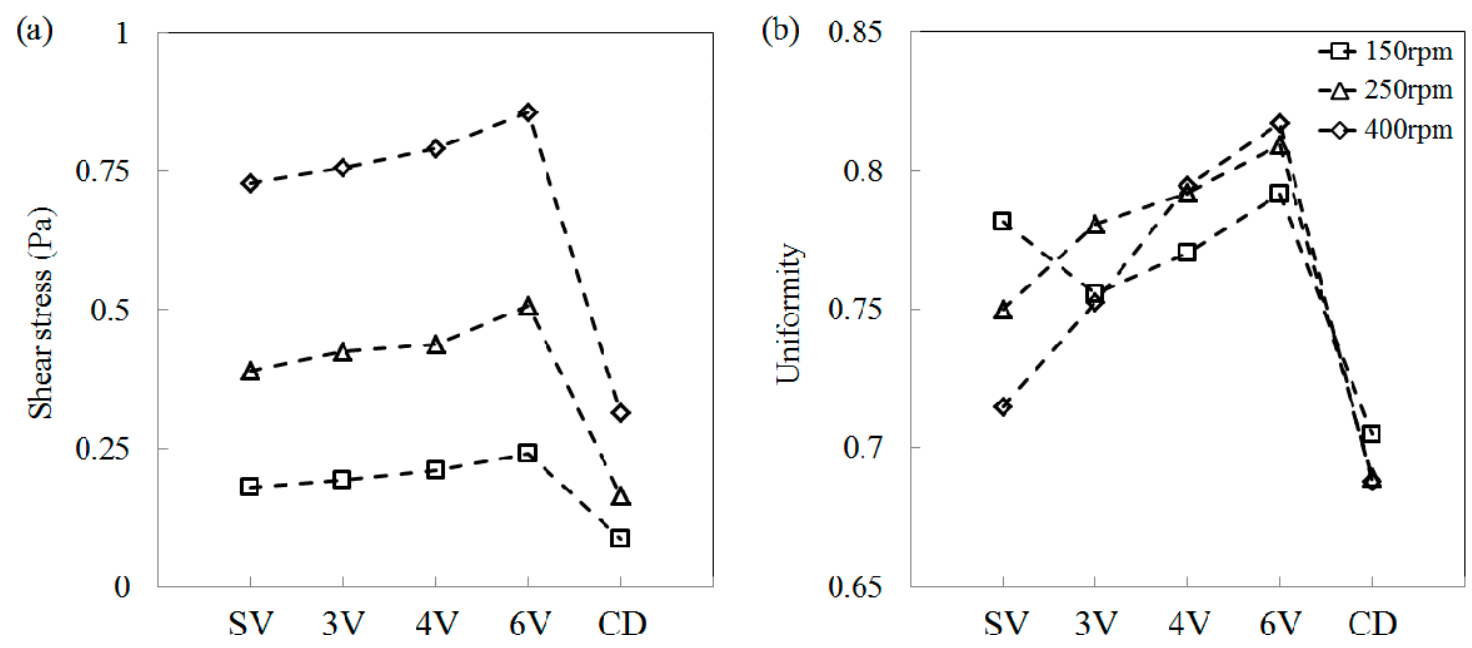

Fig. 8. Variations of facet average (a) and facet uniformity (b) of shear stress on membrane surface with respect to blade shape.

gradient. The maximum value of shear stress obtained with $400 \mathrm{rpm}$ is twice as large as that of $150 \mathrm{rpm}$. However, it seems that the increase of vane number is not an effective way to upgrade the mean shear stress, especially compared with the strategy by the increase of stirring speed. For the stirring speeds of 150, 250 and $400 \mathrm{rpm}$, the highest mean surface stress occurs always for the $6 \mathrm{~V}$ case. Instead, the increase of vane number over 6 could decrease the mean shear stress, as can be observed for the case of CD stirrer, which is be treated as an extreme case for vane number increasing.

The facet uniformity of shear stress on the membrane surface is critical for the efficient use of membrane in practical applications. A parameter for the facet uniformity of shear stress distributions is thus applied $^{[26]}$,

$r_{\tau}=1-\frac{1}{2 n} \cdot \sum_{j=1}^{n} \frac{\left|\tau_{j}-\bar{\tau}\right|}{\bar{\tau}}$,

where $\tau$ is the shear stress, $\bar{\tau}$ is the mean shear stress on a surface, and $r_{\tau}$ denotes the uniformity of shear stress distribution. Fig. 8 (b) shows the collaborating effects of the blade shapes and stirring speeds on the facet uniformity of shear stress on the membrane surface. From an overall perspective, the facet uniformity of shear stress increases as the vane number increases. Especially for $150 \mathrm{rpm}$, the facet uniformity of shear stress declines rapidly from $\mathrm{SV}$ to $3 \mathrm{~V}$, and then it increases from $3 \mathrm{~V}$ to $6 \mathrm{~V}$. It is of interest to note that the facet uniformity of shear stress for the SV stirrer is almost equal to that of $6 \mathrm{~V}$ stirrer. That is why the SV stirrer is widely used for biological separations, which only allow to be operated at low stirring speeds. Although the facet uniformity of shear stress grows with respect to the increase of vane number (from $3 \mathrm{~V}$ to $6 \mathrm{~V})$, the increase of stirring speed could accelerate its growth. It suggests that a high stirring speed need to be applied if a high uniform distribution of shear stress is requested quickly.

\section{Conclusions}

The present study focuses on the numerical investigations of blade shape effects on the flow characteristics in a stirred dead-end membrane bioreactor. The RNG $k-\varepsilon$ model based on finite volume method is applied to solve the turbulent flows using Fluent codes. The reliability and feasibility of the proposed numerical model are validated by comparisons with experimental results. Numerical implementations on the stirred internal flows were preformed, and the numerical results of flow velocities and the shear stress were well organized with careful discussions. The main findings in present study can be summarized as follows,

1) Flow structures such as wake flows and circulation loops in stirred flows were well simulated by the constructed 3D model. A region of low velocities at the membrane center is revealed. An increase of stirring speed is proposed to use to minimize the area of low velocity region.

2) The flow characteristics induced by blades with different shapes were compared with each others. Results reveal that the increase of vane number can enhance the mixing effect in flow domains. However, a circular disk stirrer is not suggested for applications, as it blocks the cylindrical reactor and goes against the formation of vertical circulations.

3) The collaborating effects of blade shapes and stirring speeds were carefully examined by analyzing the results of facet average and facet uniformity of shear stress on the membrane surface. The single vane stirrer is found to be beneficial for biological 
separations. Results also suggest that the six-vane stirrer can provide a uniform distribution of high shear stress.

However, further studies are still needed for the investigations of multi-phase flows in reactors of more complex geometry.

\section{Acknowledgments}

This work is supported by the grants from the National Natural Science Foundation of China (No. $11402084,11572203 \& 51505136)$, the Natural Science Foundation of Hunan Province (No. 2015JJ3051), the self-determined project of State Key Laboratory of Advanced Design and Manufacturing for Vehicle Body (No. 51475002) and the Fundamental Research Funds for the Central Universities (Hunan University).

\section{References}

[1] Zoh K. D., Stenstrom M. K. Application of a membrane bioreactor for treating explosives process wastewater[J]. Water research, 2002, 36(4): 1018-1024.

[2] Mutamim N. S. A., Noor Z. Z. and Hassan M. A. A. et al. Application of membrane bioreactor technology in treating high strength industrial wastewater: a performance review[J]. Desalination, 2012, 305: 1-11.

[3] Yu H. Y., Xie Y. J. and Hu M. X. et al. Surface modification of polypropylene microporous membrane to improve its antifouling property in MBR: CO 2 plasma treatment $[\mathrm{J}]$. Journal of Membrane Science, 2005, 254(1): 219-227.

[4] Lim A. L, Bai R. Membrane fouling and cleaning in microfiltration of activated sludge wastewater[J]. Journal of membrane science, 2003, 216(1): 279-290.

[5] Fadaei H., Tabaei S. R. and Roostaazad R. Comparative assessment of the efficiencies of gas sparging and backflushing to improve yeast microfiltration using tubular ceramic membranes[J]. Desalination, 2007, 217(1): 93-99.

[6] Jaffrin M. Y. Dynamic shear-enhanced membrane filtration: a review of rotating disks, rotating membranes and vibrating systems[J]. Journal of Membrane Science, 2008, 324(1): 7-25.

[7] Jaffrin M. Y. Dynamic filtration with rotating disks, and rotating and vibrating membranes: an update[J]. Current Opinion in Chemical Engineering, 2012, 1(2): 171-177.

[8] Ríos S. D., Salvadó J. and Farriol X. et al. Antifouling microfiltration strategies to harvest microalgae for biofuel[J]. Bioresource technology, 2012, 119: 406-418.

[9] Lee S. S., Burt A. and Russotti G. et al. Microfiltration of recombinant yeast cells using a rotating disk dynamic filtration system $[\mathrm{J}]$. Biotechnology and bioengineering, 1995, 48(4): 386-400.

[10] Jiang T., Zhang H. and Gao D. et al. Fouling characteristics of a novel rotating tubular membrane bioreactor[J]. Chemical Engineering and Processing: Process Intensification, 2012, 62: 39-46.

[11] Torras C., Pallarès J. and Garcia-Valls R. et al. CFD simulation of a rotating disk flat membrane module[J]. Desalination, 2006, 200(1): 453-455.

[12] Torras C., Pallares J. and Garcia-Valls R. et al. Numerical simulation of the flow in a rotating disk filtration module[J]. Desalination, 2009, 235(1): 122-138.
[13] Parvareh A., Rahimi M. and Madaeni S. S. et al. Experimental and CFD study on the role of fluid flow pattern on membrane permeate flux[J]. Chinese Journal of Chemical Engineering, 2011, 19(1): 18-25.

[14] Brou A., Ding L. and Boulnois P. et al. Dynamic microfiltration of yeast suspensions using rotating disks equipped with vanes[J]. Journal of membrane science, 2002, 197(1): 269-282.

[15] Sen D., Roy W. and Das L. et al. Ultrafiltration of macromolecules using rotating disc membrane module (RDMM) equipped with vanes: effects of turbulence promoter[J]. Journal of Membrane Science, 2010, 360(1): 40-47.

[16] Hwang K. J., Wu S. E. Disk structure on the performance of a rotating-disk dynamic filter: A case study on microalgae microfiltration[J]. Chemical Engineering Research and Design, 2015, 94: 44-51.

[17] Kim K., Jung J. Y. and Kwon J. H. et al. Dynamic microfiltration with a perforated disk for effective harvesting of microalgae[J]. Journal of Membrane Science, 2015, 475: 252-258.

[18] Sarkar A., Moulik S. and Sarkar D. et al. Performance characterization and CFD analysis of a novel shear enhanced membrane module in ultrafiltration of Bovine Serum Albumin (BSA)[J]. Desalination, 2012, 292: 53-63.

[19] Yakhot V., Orszag S. A. Renormalization group analysis of turbulence. I. Basic theory[J]. Journal of scientific computing, 1986, 1(1): 3-51.

[20] Ammar M., Chtourou W. and Driss Z. et al. Numerical investigation of turbulent flow generated in baffled stirred vessels equipped with three different turbines in one and two-stage system[J]. Energy, 2011, 36(8): 5081-5093.

[21] Tominaga Y., Stathopoulos T. Numerical simulation of dispersion around an isolated cubic building: Comparison of various types of $k-\varepsilon$ models[J]. Atmospheric Environment, 2009, 43(20): 3200-3210.

[22] Ameur H., Bouzit M. and Ghenaim A. Numerical study of the performance of multistage Scaba 6SRGT impellers for the agitation of yield stress fluids in cylindrical tanks[J]. Journal of Hydrodynamics, Ser. B, 2015, 27(3): 436442.

[23] Yataghene M., Pruvost J. and Fayolle F. et al. CFD analysis of the flow pattern and local shear rate in a scraped surface heat exchanger[J]. Chemical Engineering and Processing: Process Intensification, 2008, 47(9): 1550-1561.

[24] $\mathrm{Hu}$ X. Q., Wu P. Q. and Wang X. Y. et al. Threedimensional numerical study of flow characteristic and membrane fouling evolution in an enzymatic membrane reactor[J]. Journal of Hydrodynamics, 2016, in press.

[25] Luo J., Morthensen S. T. and Meyer A. S. et al. Filtration behavior of casein glycomacropeptide (CGMP) in an enzymatic membrane reactor: fouling control by membrane selection and threshold flux operation[J]. Journal of Membrane Science, 2014, 469: 127-139.

[26] Weltens H., Bressler H. and Terres F. et al. Optimisation of catalytic converter gas flow distribution by CFD prediction. SAE Technical Paper, 1993, No. 930780. 